\title{
Post-cholecystectomy syndrome: a retrospective study analysing the associated demographics, aetiology, and healthcare utilization
}

\author{
Saad Saleem ${ }^{1}$, Simcha Weissman ${ }^{2}$, Hector Gonzalez $^{3}$, Patricia Guzman Rojas ${ }^{4}$, Faisal Inayat ${ }^{5}$, Ali Alshati ${ }^{6}$, \\ Vinaya Gaduputi ${ }^{7}$
}

${ }^{1}$ Department of Internal Medicine, Mercy St. Vincent Medical Center, Toledo, OH, USA; ${ }^{2}$ Department of Internal Medicine, Touro College of Osteopathic Medicine, Middletown, NY, USA; ${ }^{3}$ Department of Internal Medicine, Florida Atlantic University, Boca Raton, FL, USA; ${ }^{4}$ Internal Medicine, University of Central Florida College of Medicine, Orlando, USA; ${ }^{5}$ Internal Medicine, Allama Iqbal Medical College, Lahore, Pakistan; ${ }^{6}$ Department of Internal Medicine, Maricopa Integrated Health System, Creighton University, Phoenix, AZ, USA; ${ }^{7}$ Gastroenterology, Department of Internal Medicine, St. Barnabas Hospital, health system, Bronx, NY, USA

Contributions: (I) Conception and design: S Saleem, S Weissman, H Gaduputi; (II) Administrative support: S Saleem, S Weissman, H Gaduputi; (III) Provision of study materials or patients: S Saleem; (IV) Collection and assembly of data: S Saleem; (V) Data analysis and interpretation: S Saleem; (VI) Manuscript writing: All authors; (VII) Final approval of manuscript: All authors.

Correspondence to: Saad Saleem. Department of Internal Medicine, Mercy St. Vincent Medical Center, Toledo, OH, USA.

Email: saadsaleem29@gmail.com.

Background: Post-cholecystectomy syndrome (PCS) is a group of heterogeneous signs and symptoms, predominately consisting of right upper quadrant abdominal pain, dyspepsia, and/or jaundice, manifesting after undergoing a cholecystectomy. According to some studies, as many as $40 \%$ of post-cholecystectomy patients are in fact, affected by this syndrome. This study aims to determine the demographics, aetiology, average length of hospital stay, and health care burden associated with PCS.

Methods: We queried the National Inpatient Sample (NIS) database to determine inpatient admissions of PCS between 2011 and 2014 using the ICD-9 primary diagnosis code 576.0.

Results: From 2011 to 2014, the number of inpatient admissions with a principal diagnosis of PCS totally 275. The average length of hospital stay was $4.28 \pm 4.28,3.42 \pm 2.73,3.74 \pm 1.84$, and $3.79 \pm 2.78$ days in 2011, 2012,2013 , and 2014 , respectively. The total yearly charges were $\$ 32,079 \pm \$ 24,697, \$ 27,019 \pm \$ 22,633$, $\$ 34,898.21 \pm \$ 24,408$, and $\$ 35,204 \pm \$ 32,951$ in $2011,2012,2013$, and 2014 , respectively. Notably, the primary cause of PCS in our patient sample between the year 2011 and 2014, was biliary duct dysfunction, followed by Peptic ulcer disease.

Conclusions: In conclusion, there is a strong need to examine for and treat the underlying aetiology when approaching a post-cholecystectomy patient. We found that longer hospital stays, were associated with a greater health care burden, and visa versa. Furthermore, our findings help identify at-risk populations which can contribute to improving surveillance of this costly disease.

Keywords: Post-cholecystectomy syndrome (PCS); biliary tract dysfunction; peptic ulcer disease; health care utilization.

Received: 01 May 2019; Accepted: 05 November 2019; Published: 25 October 2021.

doi: $10.21037 / \operatorname{tgh} .2019 .11 .08$

View this article at: http://dx.doi.org/10.21037/tgh.2019.11.08

\section{Introduction}

Post-cholecystectomy syndrome (PCS) is a group of heterogeneous signs and symptoms, consisting of right upper quadrant abdominal pain, dyspepsia, and/or jaundice, manifesting after undergoing a cholecystectomy. While the onset of symptoms may occur from 2 days to 25 years, the incidence of this syndrome has been reported to affect up to a staggering $40 \%$ of patients $(1,2)$. In another study, the 
incidence of recurrent symptoms among female's patients was $43 \%$, compared to $28 \%$ among male patients (3). PCS has been classified based on onset of symptoms. In early PCS - symptoms predominately occur in the postoperative period, and in late PCS—symptoms occur months to years after a cholecystectomy. The most common causes of PCS are extra-biliary in nature, such as reflex esophagitis, peptic ulcer disease, irritable bowel syndrome, or chronic pancreatitis (4). Amongst the biliary causes of PCS, they as well, can be classified into early and late. Early—or those occurring secondary to a surgical complication during a cholecystectomy, include; biliary duct injury, biliary leak, and retained cystic duct or common bile duct stones, or bile salt-induced diarrhea. Late PCS can be due to bile duct strictures, recurrent common bile duct stones, or sphincter of Oddi dysfunction. The diagnosis of PCS is primarily made clinically, based on signs and symptoms, as well as a laboratory workup-such as liver function testing. Imaging is required however, to determine the precise cause of PCS. Management is strictly tailored to treating the underlying cause. While PCS has been shown to be a common clinical phenomenon, there is relative lack of data regarding the in-patient hospitalization and health care costs associated with the diagnosis. As there are various causes of the PCS, diagnosing the exact aetiology is vital to prevent hospitalization and improve patient quality of life. This study aimed to assess the causes, frequency of hospital admissions, and health care costs, associated with a principal diagnosis of the PCS in the United States of America (USA), from 2011 to 2014.

\section{Methods}

We used the National Inpatient Sample (NIS) database to acquire data for this study. NIS is part of the Healthcare Cost and Utilization Project (HCUP) sponsored by the Agency for Healthcare Research and Quality, in Rockville, MD. The NIS is a database of hospital inpatient stays derived from billing data submitted by hospitals to state wide data organizations across the USA. It includes discharge data from more than 1,000 USA hospitals, totally almost 7 million records per year. It uses a redesigned sampling method and contains approximately $20 \%$ of non-federal hospitals. Additionally, it stratifies based on geographic region, hospital teaching status, location, and number of beds. The NIS contains hospital bill for all patients, regardless of payer, including individuals covered by Medicare, Medicaid, private insurance, and even the uninsured. The NIS database only provides administrative data for analysis; it does not provide laboratory or imaging data.

The International Classification of Diseases, Ninth Revision Clinical Modification (ICD-9-CM) diagnostic codes were used to identify the cases of post-cholecystectomy syndrome. We queried the NIS database to determine inpatient admissions of post-cholecystectomy syndrome using the ICD-9 primary diagnosis code 576.0. According to NISHCUP, primary diagnosis is a condition chiefly responsible for the hospitalization. Hence, we captured a database regarding PCS hospitalization only. The query parameters were confined for the time period between 2011 and 2014.

\section{Variables recorded}

Patient demographics included age, sex, and race. We looked at the payer status for all admissions. Length of stay is defined as the number of nights the patient stayed in the hospital. Total hospital charges are the total hospital charges for the current hospital stay. It does not cover professional or non-covered costs.

\section{Statistical analysis and the study design}

The primary outcome of the study was to assess both the frequency of hospital admissions and hospital cost, associated with the principal diagnosis of PCS. The inclusion criteria included hospital admissions with the primary diagnosis of PCS. There were no exclusion criteria.

The unit of observation is hospital visit, not patient, as an individual may have more than one hospital visit. Continuous factors are described with a mean and standard; categorical factors are described with frequency and percentages. $\mathrm{P}$ values less than 0.05 indicate a statistically significant association. All data was analysed using the IBM SPSS version 25 statistical software.

\section{Results}

From 2011 to 2014, the number of inpatient admissions with a principal diagnosis of PCS totally 275. From 2011 to 2012, there was a $38.3 \%$ decrease, from 94 to 58 diagnoses respectively (Figure 1). The length of hospital stay was $4.28 \pm 4.28,3.42 \pm 2.73,3.74 \pm 1.84$, and $3.79 \pm 2.78$ days in 2011 , 2012, 2013, and 2014, respectively (Figure 2). The total yearly charges were $\$ 32,079 \pm \$ 24,697, \$ 27,019 \pm \$ 22,633$, $\$ 34,898.21 \pm \$ 24,408$, and $\$ 35,204 \pm \$ 32,951$ in 2011,2012 , 2013, and 2014, respectively (Figure 3). There was a 


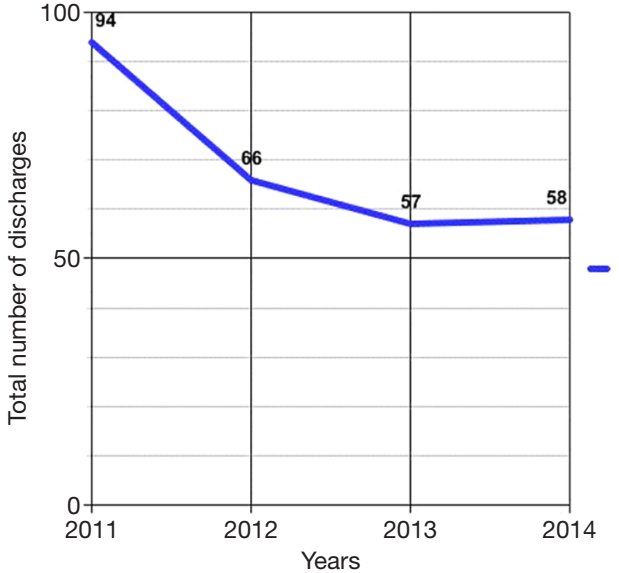

Figure 1 Total number of hospital discharges with the primary diagnosis of postcholecystectomy syndrome.

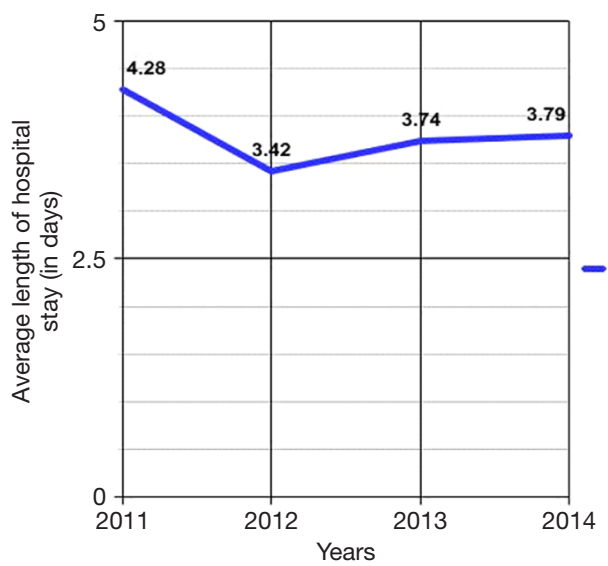

Figure 2 Average length of hospital stay (in days) over time in postcholecystectomy syndrome population.

significant decrease in the average length of hospital stay between 2011 and 2012, from 4.28 to 3.42 days $(\mathrm{P}=0.07)$, respectively. Then hospital stay started to increase again to 3.74 and 3.79 days, in 2013 and 2014, respectively. Concordantly with the decrease in average length of stay, the mean hospital charges for PCS related hospitalizations per patient decreased $18.2 \%$, from 33,017 in 2011 (adjusted for inflation), to $\$ 27,019$ in $2012(\mathrm{P}=0.18)$. There was an increase of $27.1 \%$ in hospital cost between 2012 to 2013 , from 27,449 (adjusted for inflation) to $34,898(\mathrm{P}=0.05)$, then hospital cost remained almost stable between 2013 and 2014, after adjustment for inflation occurred, from $\$ 35,449$ to $\$ 36,204$.

Patients in the age group of 30-39 years had the highest rate of hospital admissions for PCS between 2011 and 2014

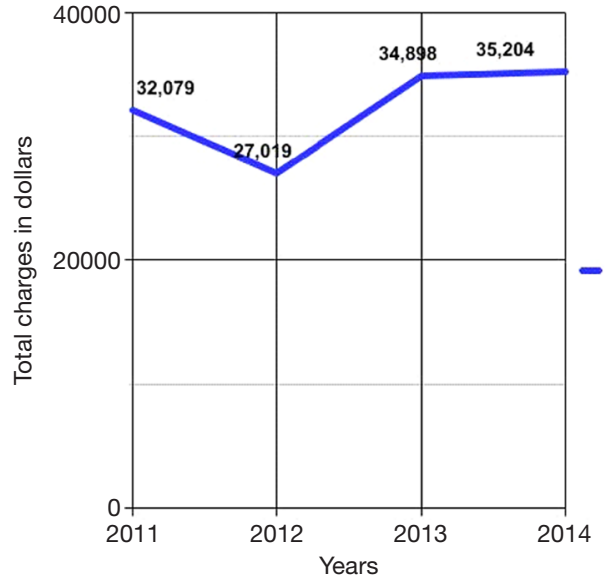

Figure 3 Average hospital charges per hospitalization over time due to postcholecystectomy syndrome.

(Table 1). The frequency of PCS for age groups 40-49, $50-59,60-69$ years is $46(16.7 \%), 43(15.6 \%)$, and 41 $(14.9 \%)$, respectively. The male to female ratio was $1: 3.5$, $61(22.2 \%)$ were male patients, whereas, $214(77.8 \%)$ were female patients. The frequency of PCS in a specific race was as follows: Caucasian-154 (56\%), Black-26 (9.5\%), Hispanic-55 (20.0\%), Asian or Pacific Islander-6 (2.2\%), Native American-2 (0.7\%), and other-9 (3.3\%). Of the 275 patients, $244(88.7 \%)$ were admitted non-electively, while $23(8.4 \%)$ were electively admitted. Out of the total 275 hospital stays, $42(15.3 \%)$ patients happened to be smokers, while only 23 (8.4\%) patients were obese.

The relative frequency of PCS was highest for private insurances-namely $106(38.5 \%)$ patients. The next highest was Medicare, followed by Medicaid, with 82 (29.8\%) and $56(20.4 \%)$ patients respectively.

While no obvious cause for PCS could be found in most patients, the recorded causes were as follows: disorders of biliary tract in $20(7.3 \%)$ patients, peptic ulcer disease accounted for 11 (4\%) patients, chronic pancreatitis 8 $(2.9 \%)$ patients, bile duct obstruction $8(2.9 \%)$ patients, and cholangitis $5(1.8 \%)$ patients. Notably, no cases of Helicobacter pylori infection, bile duct fistula or perforation, or dysfunction of the sphincter of Oddi, could be found (Table 2).

\section{Discussion}

The surgical procedure of a cholecystectomy itself is associated with many physiological changes in the gastrointestinal tract. In addition, hormonal changes primarily 
Table 1 Demographics of patients with postcholecystectomy syndrome

\begin{tabular}{|c|c|}
\hline Factor & Number (percentage) \\
\hline Total number of patients & 275 \\
\hline \multicolumn{2}{|l|}{ Age groups (years) } \\
\hline $10-19$ & $15(5.5)$ \\
\hline 20-29 & $38(13.8)$ \\
\hline 30-39 & $55(20.0)$ \\
\hline $40-49$ & $46(16.7)$ \\
\hline $50-59$ & $43(15.6)$ \\
\hline $60-69$ & $41(14.9)$ \\
\hline $70-79$ & $20(7.3)$ \\
\hline $80+$ & $17(6.2)$ \\
\hline \multicolumn{2}{|l|}{ Sex (missing 1) } \\
\hline Male & $61(22.2)$ \\
\hline Female & $214(77.8)$ \\
\hline \multicolumn{2}{|l|}{ Race (23 missing data) } \\
\hline White & $154(56)$ \\
\hline Black & $26(9.5)$ \\
\hline Hispanic & $55(20.0)$ \\
\hline Asian or Pacific Islander & $6(2.2)$ \\
\hline Native American & $2(0.7)$ \\
\hline Other & $9(3.3)$ \\
\hline Smokers & $42(15.3)$ \\
\hline Obesity & $23(8.4)$ \\
\hline \multicolumn{2}{|l|}{ Elective admission } \\
\hline Non-elective admission & $244(88.7)$ \\
\hline Elective admission & $30(10.9)$ \\
\hline \multicolumn{2}{|c|}{ Expected primary payer (1 missing data) } \\
\hline Medicare & $82(29.8)$ \\
\hline Medicaid & $56(20.4)$ \\
\hline Private including $\mathrm{HMO}$ & $106(38.5)$ \\
\hline Self-pay & $14(5.1)$ \\
\hline No charge & $2(0.7)$ \\
\hline Other & $14(5.1)$ \\
\hline
\end{tabular}

occurring in the upper gastrointestinal tract manifest after a cholecystectomy. Furthermore, the cholecysto-sphincter of Oddi reflex, cholecysto-antral reflex, and cholecysto-
Table 2 Causes of postcholecystectomy syndrome (PCS) in hospitalized patients

\begin{tabular}{lcc}
\hline Causes of PCS & Patient number & Percentage \\
\hline Peptic ulcer disease & 11 & 4.0 \\
Dyspepsia & 4 & 1.5 \\
Cholangitis & 5 & 1.8 \\
Chronic pancreatitis & 8 & 2.9 \\
Bile duct obstruction & 8 & 2.9 \\
Disorders of the biliary tract & 20 & 7.3 \\
Helicobacter pylori infection & 0 & 0 \\
Bile duct fistula & 0 & 0 \\
Bile duct perforation & 0 & 0 \\
Sphincter of Oddi dysfunction & 0 & 0 \\
\hline
\end{tabular}

esophageal reflexes are all disrupted. Therefore, there is an increased incidence of gastritis, duodenogastric reflux, and gastro-esophageal reflux-which may perhaps be the basis for the symptoms in PCS (5). Thus, the persistence of symptoms or the development of new symptoms after gallbladder removal should be no surprise.

Ultrasonography and or computed tomography (CT) scanning along with liver function testing, is the most cost efficient and productive way to initiate the workup in a patient with suspected PCS. In a retrospective study of 42 patients, Terhaar et al. concluded that although MRCP is the gold standard imaging modality, it should only be done if the common bile duct is found to be greater than $10 \mathrm{~mm}$ dilated (6). Once diagnosis is made, an ERCP can be performed to remove the stone or invasively amend the pathologic cause. As underlying pathology for PCS is diverse, sphincter of Oddi activity may be suspected. In order to evaluate sphincter of Oddi activity, the noninvasive biliary scintigraphy, has been advocated as being fairly reliable. Non-the-less, it has limited diagnostic accuracy compared with sphincter of Oddi manometry-the gold standard for assessing functional disorders as the cause of PCS (7). However, symptoms occur within weeks after a cholecystectomy, an upper gastrointestinal (GI) endoscopy is warranted, as the cause is likely to be upper GI related.

PCS can have diverse etiologies, generally differentiated into four major categories: extra-biliary, biliary, organic, and functional (8). Our results are indeed, in line, with other studies that found the main causes to be extra-biliary in nature, such as reflux esophagitis, Peptic ulcer disease, 
and dyspepsia (8-10). The most common established biliary etiologies are residual or de novo bile duct stones, with a prevalence of $5-15 \%$ (11). Other commonly found causes are; bile salt induced diarrhea/gastritis, abscess, chronic biloma, bile leak, biliary strictures, long cystic duct remnant, stenosis, and sphincter of Oddi dyskinesia $(9,10)$. Isherwood et al. found that three years post cholecystectomy was the cutoff, after which the type of aetiology changes (8). The first three years have a predominance of gastric causes, while biliary pathologies are mainly seen beyond the 3 -year mark. Skalicky et al. agreed with this temporal pattern but extended the time period from three, to 5 years, post cholecystectomy (12).

To our knowledge, in the USA, no statistically significant relationship exists between PCS and its associated demographics-namely age, sex, and race. The overall incidence has been demonstrated to be higher in females, but likely because more females undergo cholecystectomy surgeries annually. Additionally, there is no reported statistically significant relationship of PCS with age, despite symptoms being most common in the age group of 40-60 years. The same applies for race, smoking history, and BMI level, with no statistically supported relationship. Our research highlights the demographics associated with the principal diagnosis of PCS. Patients in the age group of 30-39 years had the highest rate of hospital admissions for PCS, between 2011 and 2014. The frequency of PCS for age groups $40-49,50-59,60-69$ years was $16.7 \%, 15.6 \%$, and $14.9 \%$, respectively. The male to female ratio was $1: 3.5$. Caucasians accounted for $56 \%$ of cases, while Blacks and Hispanics accounted for a combined 29.5. Out of the total 275 hospital stays, $42(15.3 \%)$ patients happened to be smokers, while only 23 (8.4\%) patients were obese. Hence, a post-cholecystectomy Caucasian female, between the ages of 30-39, complaining of right upper quadrant abdominal pain and dyspepsia, should prompt an even greater level of suspicion for PCS. Obesity and/or having a history of tobacco usage shouldn't be considered risk factors for PCS; nor by virtue arouse suspicion for this diagnosis, unless of course their symptoms necessitate a high level of suspicion.

Placed within the larger context of the overall cost of a cholecystectomy procedure, the individual healthcare burden PCS poses is indeed costly. Given that quality of care is not being compromised on any level-as only symptoms are being managed in most cases-clear opportunity exists for improved resource utilization. According to the 'healthcare value' definition-in which outcome is measured for each dollar spent-only beneficial outcomes are worthwhile (13). In our study, during the years of 2011, 2012, 2013, and 2014, the average length of hospital stay was $4.28,3.42,3.74$, and 3.79 days, respectively. Hence our data represents a clear association between length of hospital stay and financial burden.

Now that clear association between hospital stay and healthcare burden has been established, we must postulate as to what may decrease length of hospitalization. Logically, early diagnosis of post-procedure complication is one method by which costs may in fact be lowered. Not only would the decreased hospital stay not compromise special patient care, but it would even decrease the risk of infection and slower recovery rates associated with longer hospitalization. Hence early diagnosis and treatmentas opposed to symptomatic management-will shorten hospital stay and thus lower hospital costs.

In conclusion, there is a strong need amongst hospital staff and medical professionals to diagnose PCS from its onset. Our data draws light to the differential diagnoses a clinician must have when approaching a postcholecystectomy patient, as well as the proper patient demographic that should arouse a higher level of suspicion of this diagnosis. Our data also illustrates the astronomical amount of money that is allocated to provide this subset of patients with medical care. While the health care burden for PCS is considerably large, the time to diagnosis would certainly shorten the length of in-patient stay. As our data showed, the longer the hospital stay, the greater the cost associated with the diagnosis was, and visa versa. Since most patients being admitted for a chief complaint of nausea and vomiting-even post-cholecystectomy, are only treated symptomatically, the need to examine for and treat the underlying aetiology, is of paramount importance.

\section{Acknowledgments}

We are thankful for NIS-HCUP for database. Funding: None.

\section{Footnote}

Conflicts of Interest: All authors have completed the ICMJE uniform disclosure form (available at http://dx.doi. org/10.21037/tgh.2019.11.08). The authors have no conflicts of interest to declare.

Ethical Statement: The authors are accountable for all aspects of the work in ensuring that questions related 
to the accuracy or integrity of any part of the work are appropriately investigated and resolved. The study was conducted in accordance with the Declaration of Helsinki (as revised in 2013). Institutional ethical approval was waived.

Open Access Statement: This is an Open Access article distributed in accordance with the Creative Commons Attribution-NonCommercial-NoDerivs 4.0 International License (CC BY-NC-ND 4.0), which permits the noncommercial replication and distribution of the article with the strict proviso that no changes or edits are made and the original work is properly cited (including links to both the formal publication through the relevant DOI and the license). See: https://creativecommons.org/licenses/by-nc-nd/4.0/.

\section{References}

1. Yamada T. editor. Textbook of gastroenterology. 2nd ed. Philadelphia: Lippincott; 1995, chapter 104.

2. Zhou PH, Liu FL, Yao LQ, et al. Endoscopic diagnosis and treatment of postcholecystectomy syndrome. Hepatobiliary Pancreat Dis Int 2003;2:117-20.

3. Bodvall B, Overgaard B. Cystic duct remnant after cholecystectomy: incidence studied by cholegraphy in 500 cases, and significance in 103 reoperations. Ann Surg 1966;163:382-90.

4. Rogy MA, Fugger R, Herbst F, et al. Reoperation after cholecystectomy. The role of the cystic duct stump. HPB Surg 1991;4:129-34.

5. Shirah BH, Shirah HA, Zafar SH, et al. Clinical patterns

doi: $10.21037 / \operatorname{tgh} .2019 .11 .08$

Cite this article as: Saleem S, Weissman S, Gonzalez H, Rojas PG, Inayat F, Alshati A, Gaduputi V. Post-cholecystectomy syndrome: A retrospective study analysing the associated demographics, aetiology, and healthcare utilization. Transl Gastroenterol Hepatol 2021;6:58. of postcholecystectomy syndrome. Ann Hepatobiliary Pancreat Surg 2018;22:52-7.

6. Terhaar OA, Abbas S, Thornton FJ, et al. Imaging patients with "post-cholecystectomy syndrome": an algorithmic approach. Clin Radiol 2005;60:78-84.

7. Craig AG, Peter D, Saccone GT, et al. Scintigraphy versus manometry in patients with suspected biliary sphincter of Oddi dysfunction. Gut 2003;52:352-7.

8. Isherwood J, Oakland K, Khanna A. A systematic review of the aetiology and management of post cholecystectomy syndrome. Surgeon 2019;17:33-42.

9. Madacsy L, Dubravcsik Z, Szepes A. Postcholecystectomy syndrome: from pathophysiology to differential diagnosis - a critical review. Pancreat Disord Ther 2015;5:162.

10. Jaunoo SS, Mohandas S, Almond LM. Postcholecystectomy syndrome (PCS). Int J Surg 2010;8:15-7.

11. Zhu JG, Zhang ZT. Laparoscopic remnant cholecystectomy and transcystic common bile duct exploration for gallbladder/cystic duct remnant with stones and choledocholithiasis after cholecystectomy. J Laparoendosc Adv Surg Tech A 2015;25:7-11.

12. Skalicky M. Dynamic changes of echogenicity and the size of the papilla of vater before and after cholecystectomy. J Int Med Res 2011;39:1051-62.

13. Brauer DG, Hawkins WG, Strasberg SM, et al. Cost variation in a laparoscopic cholecystectomy and the association with outcomes across a single health system: implications for standardization and improved resource utilization. HPB (Oxford) 2015;17:1113-8. 